\title{
Multiwavelength detectability of isolated black holes in the Milky Way
}

\author{
Francesca Scarcella, ${ }^{1 \star}$ Daniele Gaggero ${ }^{\oplus},{ }_{1}^{1}$ Riley Connors ${ }^{๑},{ }^{2}$ Julien Manshanden, ${ }^{3}$ Massimo Ricotti ${ }^{\oplus 4}$ \\ and Gianfranco Bertone ${ }^{5}$ \\ ${ }^{1}$ Instituto de Física Teórica UAM-CSIC, Campus de Cantoblanco, E-28049 Madrid, Spain \\ ${ }^{2}$ Cahill Center for Astronomy and Astrophysics, California Institute of Technology, Pasadena, CA 91125, USA \\ ${ }^{3}$ II. Institut für Theoretische Physik, Universität Hamburg, D-22761 Hamburg, Germany \\ ${ }^{4}$ Department of Astronomy, University of Maryland, College Park, MD 20740, USA

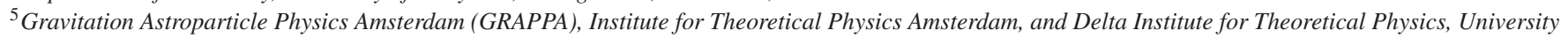 \\ of Amsterdam, Science Park 904, NL-1098 XH Amsterdam, the Netherlands
}

Accepted 2021 May 20. Received 2021 April 27; in original form 2020 December 21

\begin{abstract}
Isolated black holes in our Galaxy have eluded detection so far. We present here a comprehensive study on the detectability of isolated stellar-mass astrophysical black holes that accrete interstellar gas from molecular clouds in both the local region and the Central Molecular Zone. We adopt a state-of-the-art model for the accretion physics backed up by numerical simulations, and study the number of observable sources in both the radio and X-ray band, as a function of a variety of parameters. We discuss in particular the impact of the astrophysical uncertainties on our prediction for the number of bright X-ray sources in the central region of the Galaxy. We finally consider future developments in the radio domain and assess the potential of SKA to detect a population of astrophysical black holes accreting gas in our Galaxy.
\end{abstract}

Key words: astroparticle physics - black hole physics - ISM: jets and outflows.

\section{INTRODUCTION}

A large population of Stellar-mass black holes (BHs) is believed to exist in our Galaxy: several works (Shapiro \& Teukolsky 1983; Samland 1998; Caputo et al. 2017) agree on an order-of-magnitude estimate of $\sim 10^{8}$ BHs produced by the collapse of massive stars in the Galaxy. This large number of BHs has eluded detection so far, with the exception of $\simeq 60$ objects $^{1}$ that are part of binary systems and accrete a significant amount of mass from a companion star: such systems are known as black hole X-ray binaries (BH-XRBs) and appear as bright $\mathrm{X}$-ray sources in the sky.

Here, we focus on isolated BHs, whose detection represents a major challenge in modern astronomy. The problem has been discussed in several recent studies focused on the future detectability of the multiwavelength radiation possibly emitted by these objects when they accrete matter from interstellar clouds either in the vicinity of the Sun (Maccarone 2005; Fender, Maccarone \& Heywood 2013) or in the regions near the Galactic Centre, as recently discussed in Tsuna, Kawanaka \& Totani (2018) and Tsuna \& Kawanaka (2019). The latter region seems particularly promising. In fact, an extensively studied complex of giant molecular clouds usually called Central Molecular Zone (CMZ) characterizes a vast region that extends up to $\simeq 200$ pc away from the Galactic Centre, and provides a huge reservoir of molecular hydrogen. The highdensity clumps within these region appear as ideal targets for

\footnotetext{
^E-mail: francesca.scarcella@uam.es

${ }^{1}$ http://www.astro.puc.cl/BlackCAT/
}

astronomical searches of compact objects based on gas accretion.

In order to perform these analyses, it is crucial to develop an accurate modelling of (i) the accretion process of baryonic matter on to a compact object in order to compute the expected accretion rate as function of the environmental conditions and the $\mathrm{BH}$ mass and speed; and (ii) the non-thermal spectrum of the radiation emitted by the accreted gas in different wavelengths, from radio all the way up to the hard X-ray band.

Regarding the former aspect, a simplified model for the accretion physics is adopted in the aforementioned papers, based on a rescaling of the Bondi-Hoyle-Lyttleton (BHL) formula. However, the accretion problem exhibits a richer phenomenology. Radiation feedback plays a crucial role, and is expected to shape the surrounding environment in a remarkable way, forming a cometaryshaped ionized region around the compact object. A state-of-the-art treatment of accretion in the presence of radiative feedback, backed up by numerical simulations, was presented in a series of works (Park \& Ricotti 2011, 2012; Park \& Ricotti 2013, PR13 hereafter; Sugimura \& Ricotti 2020) and showed a more complicated behaviour of the accretion rate with respect to the $\mathrm{BH}$ speed compared to the BHL scaling. This model features an increase at low velocities, and an asymptotic decrease to the BHL rate at large velocities. The PR13 results were recently applied to compact object searches in the context of the quest for BHs of primordial origin (Manshanden et al. 2019). However, a comprehensive study of this model related to the population of astrophysical BHs is still lacking.

As for the prescription for the multiwavelength (radio/X-ray) nonthermal emission, the absence of any observational detection of 
isolated accreting stellar-mass BHs means any physical model one can develop carries large uncertainties. However, one can utilize the vast knowledge gathered in the studies of both accreting BH-XRBs and accreting supermassive BHs to inform such a prescription. In particular, the Fundamental Plane relation between radio and $\mathrm{X}$ ray emission (Plotkin et al. 2012) derived from the study of the aforementioned systems can be a precious tool to characterize the relation between radio and $\mathrm{X}$-ray emission.

The aim of this paper is to characterize the detection prospect of a population of astrophysical BHs in view of the future development of radio and X-ray astronomy (with particular reference to the SKA experiment for the former), taking into account the state-of-the-art accretion formalism from PR13.

The paper is organized as follows: In Sections 2 and 3, we review the main aspects of accretion physics with particular focus on the PR13 model and discuss the emission mechanisms that are most relevant in the radio and X-ray domain. Then, we present our methodology in Sections 4 and 5 and turn our attention to the astrophysical BHs in the vicinity of the Sun in Section 6, estimating the number of detectable X-ray sources. We then focus on the Central Molecular Zone in Section 7 and present a comprehensive parametric study of the expected X-ray emission from astrophysical BHs in this region. Finally, in Section 8, we present a forecast about the number of radio sources potentially detectable by SKA in association with the population of isolated astrophysical $\mathrm{BHs}$ accreting gas in the CMZ.

\section{SETTING THE STAGE: STATE-OF-THE-ART ACCRETION PHYSICS}

In this section, we describe in detail the accretion model introduced in PR13 and compare it to the textbook BHL model.

\subsection{The BHL model}

According to the BHL accretion model (Hoyle \& Lyttleton 1939; Bondi \& Hoyle 1944), the rate of accretion on to an isolated compact object of mass $M$ moving at a constant speed $\mathrm{v}_{\mathrm{BH}}$ is given by

$\dot{M}_{\mathrm{BHL}}=4 \pi \frac{(G M)^{2} \rho}{\left(\mathrm{v}_{\mathrm{BH}}^{2}+c_{\mathrm{s}}^{2}\right)^{3 / 2}}$,

where $\rho$ and $c_{\mathrm{s}}$ are, respectively, the density and the sound speed that characterize the ambient medium and $G$ is the gravitational constant. This formula is usually re-scaled by introducing a suppression factor $\lambda$. Fender et al. (2013), for instance, found that values larger than $\lambda=0.01$ are excluded, under realistic assumptions, by observations of the local region, where a significant population of isolated $\mathrm{BHs}$ should be present. Tsuna et al. (2018) and Tsuna \& Kawanaka (2019) consider values between $\lambda=0.1$ and $\lambda=10^{-3}$. The $\lambda$ parameter may effectively capture the outflow of material that is expelled from the Bondi sphere, as suggested by several authors (Blandford \& Begelman 1999). Its introduction is also supported by the non-observation of a large population isolated neutron stars in the local region (Perna et al. 2003) and the studies of nearby active galactic nuclei (AGNs; Pellegrini 2005) as well as the supermassive $\mathrm{BH}$ at the Milky Way centre, Sagittarius A* (Baganoff et al. 2003). In conclusion, it seems that the BHL accretion formula may overestimate the accretion rate by orders of magnitude, although the physical mechanism behind this deviation is still disputed. One such mechanism is introduced in PR13, as we discuss below.

\subsection{The Park-Ricotti model}

The hydrodynamical simulations performed in PR13 show that, when radiative feedback is taken into account, a cometary-shaped ionized region is formed around the $\mathrm{BH}$, as it moves through the interstellar medium. The model proposed in the same work combines the BHL formula with the modelling of the ionization front to obtain an analytical formula in agreement with the results of the simulations. We review it here.

BHL accretion is assumed to hold within the ionized region, such that the PR13 accretion rate, can be written as

$\dot{M}_{\mathrm{PR} 13}=4 \pi \frac{(G M)^{2} \rho_{\mathrm{in}}}{\left(\mathrm{v}_{\mathrm{in}}^{2}+c_{\mathrm{s}, \mathrm{in}}^{2}\right)^{3 / 2}}$,

where $c_{\mathrm{S} \text {, in }}$ and $\rho_{\text {in }}$ are the sound speed and density of the ionized medium, and $v_{\text {in }}$ is its velocity relative to the $\mathrm{BH}$. The sound speed $c_{\mathrm{s}, \text { in }}$ is a free parameter of the model (of the order of few tens of $\mathrm{km} \mathrm{s}^{-1}$ ). Applying Euler's equations to the ionization front, $\rho_{\text {in }}$ and $\mathrm{V}_{\text {in }}$ can be expressed in terms of the corresponding quantities of the external neutral medium: its sound speed $c_{s}$, its density $\rho$, and the relative velocity of the $\mathrm{BH}, \mathrm{v}_{\mathrm{BH}}$. This step is left to the Appendix.

A crucial feature of the ionization front arises for velocities in the range $\mathrm{v}_{\mathrm{D}} \leq \mathrm{v}_{\mathrm{BH}} \leq \mathrm{v}_{\mathrm{R}}$, where

$\mathrm{v}_{\mathrm{R}} \approx 2 c_{\mathrm{s}, \mathrm{in}}$,
$\mathrm{v}_{\mathrm{D}} \approx \frac{c_{\mathrm{s}}^{2}}{2 c_{\mathrm{s}, \mathrm{in}}}$.

In this velocity range, the pressure wave building behind the ionization front detaches from it as a shock into the neutral material (in short, the ionization front becomes D-type). A dense cometaryshaped shell forms upstream the BH. Part of the flux of matter is deviated and the density and velocity of the gas beyond the shock are reduced significantly. Even if the $\mathrm{BH}$ moves at supersonic velocities, the gas velocity behind the shell is of the order of the speed of sound (hence the flow of matter is stopped by the bow-shock/shell). In this regime, the hot ionized region surrounding the $\mathrm{BH}$ becomes significantly underdense compared to the density of the neutral medium. Since the accretion rate is proportional to the gas density $\rho_{\text {in }}$, this results in a strong suppression of the former. Furthermore, it can be shown that, in the regime where the shock is present, the density inside the ionized region increases with the BH speed. This implies an accretion that increases with velocity, an opposite behaviour with respect to the BHL scaling. More precisely, for $\mathrm{v}_{\mathrm{D}} \leq \mathrm{v}_{\mathrm{BH}} \leq \mathrm{v}_{\mathrm{R}}$, we obtain

$\dot{M}_{\mathrm{PR} 13}=\pi \frac{(G M)^{2} \rho\left(\mathrm{v}_{\mathrm{BH}}^{2}+c_{\mathrm{s}}^{2}\right)}{\sqrt{2} c_{\mathrm{s}, \mathrm{in}}^{5}}$,

which increases quadratically with the $\mathrm{BH}$ velocity, in sharp contrast to the behaviour of the BHL rate, which presents $a \propto v_{B H}^{-3}$ dependence.

The velocity dependence of the BHL rate is recovered in the highvelocity regime, $\mathrm{v}_{\mathrm{BH}}>\mathrm{v}_{\mathrm{R}}$, when the shock is not present. In this regime, the densities and velocities of the gas on the two sides of the ionization front are similar. This in turn implies an accretion rate similar to the BHL rate.

The complete velocity dependence of the PR13 rate is shown in Fig. 1, for varied gas densities, BH masses, and sound speeds of the ionized region. For comparison, the BHL rate with $\lambda=1$ is also shown. We can observe in this figure how the BHL rate decreases monotonically with velocity, whereas the PR13 rate peaks at $\mathrm{v}_{\mathrm{BH}}=$ $\mathrm{v}_{\mathrm{R}}=2 c_{\mathrm{s} \text {,in }}$ and is suppressed at lower velocity, as a consequence of the formation of the bow shock. 

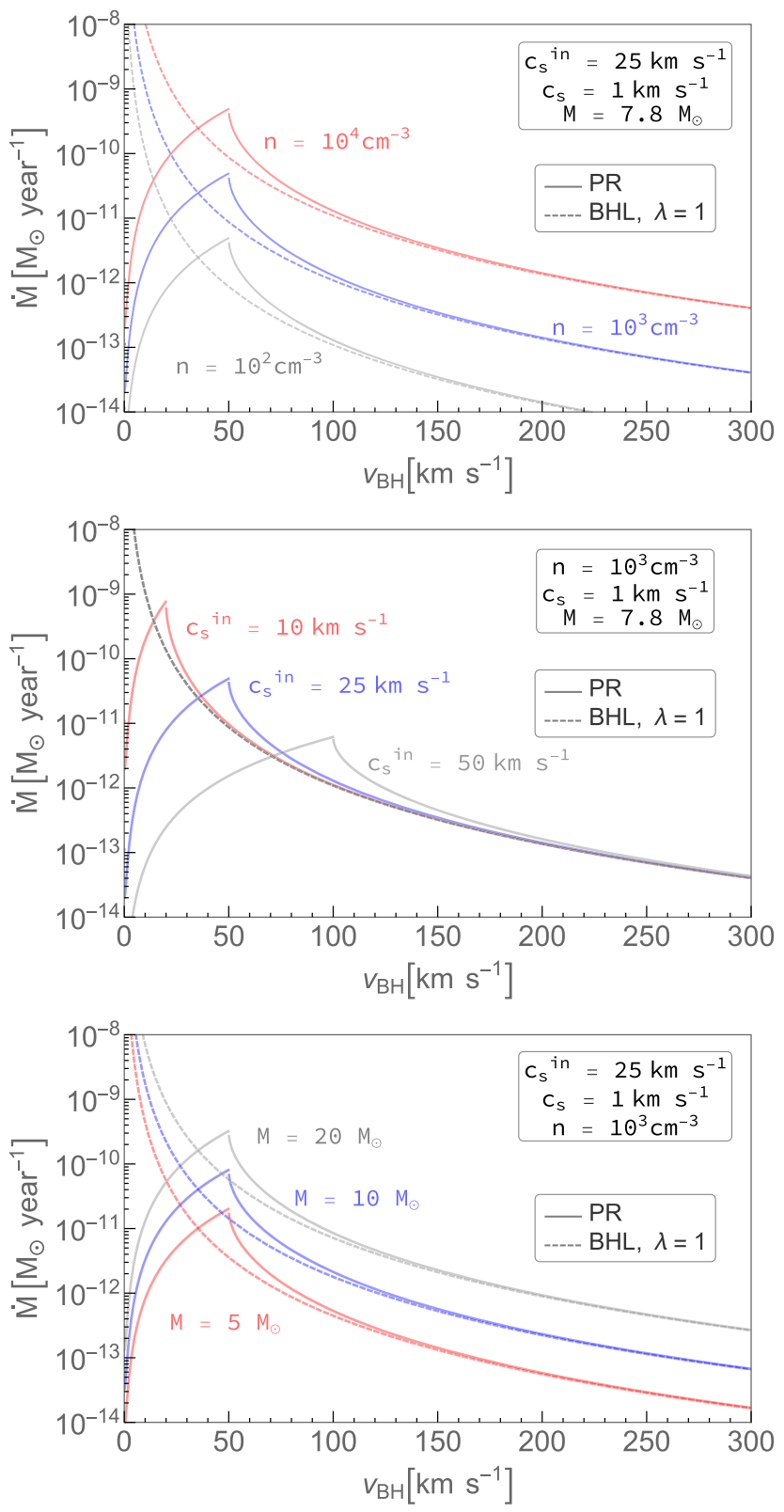

Figure 1. Accretion rate as a function of the $\mathrm{BH}$ speed. We show the accretion rate obtained according to the PR13 and BHL models, as a function of the $\mathrm{BH}$ speed and other relevant parameters. For the BHL rate, we set the suppression factor $\lambda=1$ to allow for a more direct comparison. The two models agree at high velocity, but predictions differ by many orders of magnitude in the low velocity range.

For $v<\mathrm{v}_{\mathrm{D}}$, the rate increases again. However, notice that this transition typically happens at velocities of $\approx 0.1 \mathrm{~km} \mathrm{~s}^{-1}$ (see equation A4), which are of little relevance for this work, and is not visible in Fig. 1.

The different velocity dependence of the PR13 rate compared to the BHL rate has important consequences when studying the emission properties of a $\mathrm{BH}$ population characterized by a given velocity distribution. According to the BHL prescription, the lowvelocity tail of the population is the easiest to detect. Following PR13, the highest emissions are instead associated with BHs with intermediate velocities.
Furthermore, BHL can predict very high accretion rates if the speeds are low enough, which can easily lead to overshooting experimental bounds, while the highest rates predicted by PR13 are orders of magnitude smaller.

As a final remark, using equation (4) to express the peak of the PR13 rate in terms of the Eddington accretion rate $\dot{M}_{\text {Edd }}$ :

$$
\frac{\left.\dot{M}_{\mathrm{PR} 13}\right|_{\mathrm{v}_{\mathrm{BH}}=\mathrm{v}_{\mathrm{R}}}}{\dot{M}_{\mathrm{Edd}}} \approx 10^{-4}\left(\frac{M_{\mathrm{BH}}}{10 \mathrm{M}_{\odot}}\right)\left(\frac{\rho / m_{p}}{10^{3} \mathrm{~cm}^{-3}}\right)\left(\frac{c_{s, \text { in }}}{25 \mathrm{~km} \mathrm{~s}^{-1}}\right)^{-3},
$$

one can see that the accretion rate will always be highly subEddington in the range of $\mathrm{BH}$ masses $M_{\mathrm{BH}}$, gas densities $\rho$, and ionized sound speeds $c_{s}$, in we consider in this work. This is taken into account when defining the prescription for the luminosity in the next section.

\section{EMISSION MECHANISMS}

In order to translate the predicted accretion rates of a population of isolated BHs into a prediction of detectable sources, we need estimates of the associated bolometric luminosity of a given source, and the spectral energy distribution (SED). Our focus is on the X-ray and radio luminosity, $L_{\mathrm{X}}$ and $L_{\mathrm{R}}$, of the accreting $\mathrm{BH}$. We can estimate both by considering the SED of the $\mathrm{BH}$ at varying accretion rates. We are particularly interested in emission from highly sub-Eddington accreting BHs (as predicted by equation 5). Therefore, in this section, we describe our simple framework of the properties of sub-Eddington flows on to isolated $\mathrm{BHs}$ based on our prior understanding of known weak accretors in nature: Galactic BH-XRBs and low-luminosity AGN.

Radiatively inefficient accretion flow (RIAF) models have spearheaded many studies regarding the emission mechanisms associated with such sub-Eddington accretion flows, with a focus both on Galactic BH-XRBs at low accretion rates, and the supermassive BH in the Galactic Centre, Sgr A* (Narayan \& Yi 1994; Yuan, Quataert \& Narayan 2003).

The regularly cited RIAF models that first considered the emission processes in inefficiently radiating accreting $\mathrm{BHs}$ are classed as advection-dominated accretion flows (ADAFs; Narayan \& Yi 1994; Esin, McClintock \& Narayan 1997). In the ADAF model, Bremsstrahlung radiation dominates the observed spectrum at the lowest accretion rates, and thus the spectrum has some curvature and its peak energy depends on the thermal gas temperature. As the accretion rate and gas density increase, Inverse Compton (IC) scattering begins to dominate (likely via SSC, i.e. Synchrotron photons become scattered), though if densities remain low enough, the spectrum will still show come curvature. At higher accretion rates, the IC scattering process is more efficient, resulting in a power-lawlike spectrum in the X-ray band.

The key thermodynamic property of RIAFs is the inefficient cooling of ions due to the Coulomb decoupling of electrons and ions in the accreting, low-density plasma. Due to this decoupling, such flows are likely well described by a two-temperature electron-ion plasma, with only electrons radiating via Bremsstarhlung, Synchrotron, and inverse Compton scattering (Esin et al. 1997). The models, as well as a plethora of observational evidence, show that such flows display radiative inefficiency, with $\eta=0.1 \dot{M} / \dot{M}_{\text {crit }}$, where $\eta$ is defined by

$L=\eta \dot{M}$,

and $\dot{M}_{\text {crit }}$ is the accretion rate below which we have an RIAF. We assume $\dot{M}_{\text {crit }}$ is the accretion rate corresponding to 1 per cent of the 
Eddington luminosity with $\eta_{\text {Edd }}=0.1$ (Fender et al. 2013). The bolometric luminosity $L$ in such inefficient states is thus given by

$L=0.1 \frac{\dot{M}^{2}}{\dot{M}_{\text {crit }}}$,

thus exhibiting a quadratic scaling $L \propto \dot{M}^{2}$ (Esin et al. 1997), as opposed to the linear behaviour observed at higher efficiency.

A significant fraction of this bolometric luminosity is typically assumed to fall in the X-ray band. Following Fender et al. (2013), we assume a $L_{X} / L$ fraction of $\simeq 30$ per cent, allowing for a direct calculation of the X-ray luminosity from equation (7).

Radiatively inefficient Galactic BH-XRBs also almost ubiquitously launch outflows. Such outflows are typically in the form of steady, self-absorbed relativistic jets (Fender 2001). Ballistic, transient jets are also observed during spectral state transitions at higher accretion rates. Such jets are typically identified via their radio emission, and are shown to be present during quiescence, at luminosities below $10^{-8} L_{\text {Edd }}$ (Plotkin et al. 2015). These steady jets exhibit a flat-to-inverted spectrum from radio through to IR frequencies - a consequence of self-absorbed synchrotron emission through the optically thick regions of the jet (see e.g. Markoff, Nowak \& Wilms 2005).

In addition, multiple studies have now established that the luminosity of such radio-emitting jets scales in a consistent and predictable way with the $\mathrm{X}$-ray emitting plasma in the inner regions of the flow. This scaling, $L_{\mathrm{R}} \propto L_{\mathrm{x}}^{0.7}$ (Corbel et al. 2000, 2003; Gallo, Fender \& Pooley 2003; Corbel, Koerding \& Kaaret 2008; Miller-Jones et al. 2011; Gallo et al. 2014), is known as the radio-X-ray correlation, and applies to Galactic BH-XRBs (though other compact objects have been tracked in this phase space, showing similar but distinct trends of their own). Therefore, by using this analogy between isolated BHs and their low-accretion rate counterparts in binaries, we can infer that isolated BHs will similarly launch these steady jets, and thus their radio and $\mathrm{X}$-ray luminosities will scale according to this radiatively inefficient track we observe in Galactic BH-XRBs.

However, in order to invoke a mass scaling and capture lowluminosity accretion on to a mass-variable population of isolated $\mathrm{BHs}$, one must refer to the established connection between Galactic BH-XRBs and AGN - the Fundamental Plane (FP) of black hole activity (Merloni, Heinz \& di Matteo 2003; Falcke, Körding \& Markoff 2004; Plotkin et al. 2012). The FP is, in a sense, an extension of the radio-X-ray correlation discovered in Galactic BH-XRBs to their supermassive counterparts. The FP is an empirical, parametrized relation between the X-ray luminosities, radio luminosities, and masses of hard state Galactic BH-XRBs with steady jets, and AGN of types that display similar X-ray emission characteristics. These include low-luminosity AGN (LLAGN), low-ionization nuclear emission-line regions (LINERS), Faranoff-Riley type I (FRI), and BL Lacs. It is understood that the fundamental connection between these types of AGN and Galactic BH-XRBs is simply their subEddington accretion rates, and the presence of radio-emitting jets. All sources in the FP display something resembling a power-law spectrum in the X-ray band and a flat-to-inverted radio spectrum.

To determine the radio emission, we invoke this scaling relation, thus assuming that an isolated population of low-luminosity accreting BHs will display similar spectral properties as the aforementioned systems. In particular, this thus relies on the assumption that the BHs will launch jets. We remark that this assumption (and the presence of an accretion disk on small scales) is not incompatible with a spherical accretion pattern on large scales.
We adopt the scaling relation determined in one of the most recent such FP studies (Plotkin et al. 2012):

$$
\begin{aligned}
\log \left(\frac{L_{\mathrm{X}}}{\mathrm{erg} \mathrm{cm}^{-2} \mathrm{~s}^{-1}}\right)= & (1.45 \pm 0.04) \log \left(\frac{L_{\mathrm{R}}}{\mathrm{erg} \mathrm{cm}^{-2} \mathrm{~s}^{-1}}\right) \\
& -(0.88 \pm 0.06) \log \left(\frac{M_{\mathrm{BH}}}{\mathrm{M}_{\odot}}\right) \\
& -6.07 \pm 1.10,
\end{aligned}
$$

where $L_{X}$ is the $\mathrm{X}$-ray luminosity in the $2-10 \mathrm{keV}$ band and $L_{R}$ is the radio luminosity at $5 \mathrm{GHz}$. Thus, we have a simple prescription for both the X-ray luminosity, $L_{\mathrm{X}}$, and radio luminosity, $L_{\mathrm{R}}$, of an isolated accreting $\mathrm{BH}$.

\section{ESTIMATING THE NUMBER OF VISIBLE SOURCES}

Having described our prescription for the accretion rate and the associated non-thermal emission, we now turn at assessing the number of isolated astrophysical BHs that are potentially detectable by the current (and forthcoming) generation of X-ray (with particular focus on the hard X-ray band) and radio experiments. In this section, we summarize the main points of our methodology. Our main observable is the number of sources associated with a radiation flux above detection threshold, i.e. that satisfy $\phi>\phi^{*}$, where $\phi$ is the flux at Earth defined as $\phi=L /\left(4 \pi r^{2}\right)$ with $r$ the distance to the source from Earth, and $\phi^{*}$ is the threshold value.

Previous studies (Fender et al. 2013; Tsuna et al. 2018; Tsuna \& Kawanaka 2019) obtained their estimates through Monte Carlo simulations of a BH population. Here, we adopt instead a semianalytical approach that allows us to perform a comprehensive parametric study associated with the physical model of accretion physics described above.

Here, we describe the general prescription for computing the number of visible sources, which we will apply with some variation to the two regions of interest for this work: the local region (Section 6) and the central molecular zone (CMZ, Section 7). We obtain the probability for a $\mathrm{BH}$ to emit above threshold by integrating the joint pdf of the relevant randomly distributed variables over the volume defined by the condition $\phi>\phi^{*}$.

Regarding the $\mathrm{BH}$ population, the random variables entering the flux expression are speed, mass, and distance from Earth. We neglect any possible correlation between these variables. In addition, we also treat as a random variable the density of the insterstellar medium at each BH location. In both analysis we present below, we make the assumption that the gas density and $\mathrm{BH}$ position are not correlated. Therefore, we obtain the expected number of luminous sources $N_{\text {sources }}$ by computing the following integral:

$$
\begin{aligned}
& N^{\text {sources }}\left(\phi^{*},\left\{p_{i}\right\},\left\{q_{i}\right\}\right) \\
& =N^{\mathrm{tot}} \int_{\phi\left(\mathrm{v}_{\mathrm{BH}}, M, d,\left\{p_{i}\right\}\right)>\phi^{*}} P\left(\mathrm{v}_{\mathrm{BH}}\right) P(M) P(r) P(n) . \\
& \quad \times \mathrm{dv}_{\mathrm{BH}} \mathrm{d} M \mathrm{~d} r \mathrm{~d} n
\end{aligned}
$$

Here, $P\left(\mathrm{v}_{\mathrm{BH}}\right), P(M), P(r)$, and $P(n)$ are the normalized pdfs describing, respectively, the $\mathrm{BH}$ speed, mass, distance from Earth and the interstellar medium density at its location. With $\left\{p_{i}\right\}$ we indicate the free parameters entering the expression for the flux, and which we consider to be fixed for all BHs. These are the sound speed of the neutral medium $c_{s}$, the sound speed in the ionized region $c_{s}^{\text {in }}$, the fraction of bolometric luminosity $L_{x} / L$ and, in the case of BHL accretion, the suppression factor $\lambda$ (regarding the first of these, we remark that variations of the sound speed of the neutral medium 
$c_{s}$ have a negligible impact on the flux magnitude). Finally, the parameters that define the pdfs for the random variables, which we indicate with $\left\{q_{i}\right\}$, should also be regarded as free parameters of the model. We discuss them in the next section.

\section{CHARACTERIZING THE BH POPULATION}

\subsection{Mass}

We assume $\mathrm{BH}$ masses to follow a normal distribution of mean $\mu_{\text {mass }}=7.8 \mathrm{M}_{\odot}$ and $\sigma_{\text {mass }}=1.2 \mathrm{M}_{\odot}$. This distribution was obtained from the study of X-ray binaries by Özel et al. (2010) and confirmed in an independent analysis by Farr et al. (2011). It has also been employed by previous studies on this topic such as the works of Fender et al. (2013) and Tsuna et al. (2018).

\subsection{Speed}

The $\mathrm{BH}$ velocity is given by the combination of two components: the velocity of the progenitor star, $\mathrm{v}_{\mathrm{star}}$, and the kick the $\mathrm{BH}$ receives at birth due to the supernova explosion, $\mathrm{v}_{\mathrm{kick}}$. As for the former, we are concerned with the velocities relative to the molecular gas, so we ignore the rotational component along the Galactic disk and consider exclusively the velocity dispersion $\sigma_{\mathrm{D}}$. This is well measured for both nearby stars and stars in the Galactic Centre.

On the other hand, little is known about the magnitude of the natal BH kicks. A study of pulsars proper motions by Hobbs et al. (2005) concluded that neutron star kicks obey a Maxwell Boltzmann distribution with $\sigma=265 \mathrm{~km} \mathrm{~s}^{-1}$. Re-scaled with the average masses (Fender et al. 2013), this gives $\sigma_{\text {kick }} \approx 50 \mathrm{~km} \mathrm{~s}^{-1}$, corresponding to a mean of $\mu_{\text {kick }} \approx 75 \mathrm{~km} \mathrm{~s}^{-1}$. We consider here as reference values $\mu_{\text {kick }}=50 \mathrm{~km} \mathrm{~s}^{-1}$ ('low kick') and $\mu_{\text {kick }}=100 \mathrm{~km} \mathrm{~s}^{-1}$ ('high kick').

The BH speed, resulting from the combination of these two independent components, is then distributed following a Maxwell Boltzmann of mean: $\mu_{\mathrm{BH}}=\sqrt{\mu_{\text {star }}^{2}+\mu_{\text {kick }}^{2}}$.

Hence, the uncertainties in both the kick and the progenitor star's velocity dispersion are enclosed in only one parameter, $\mu_{\mathrm{BH}}$.

\subsection{Distance from Earth}

We will assume the BHs to be distributed uniformly in both regions under analysis and derive the distance distribution accordingly.

\subsection{Density of the interstellar medium}

The interstellar medium is usually described as composed by three different components: the ionized component, the neutral gas, and the molecular clouds. In the PR13 picture, the probability of obtaining large enough fluxes from a $\mathrm{BH}$ found in the first two of these components turns out to be negligible. We will therefore consider only densities associated with molecular clouds.

\section{SEARCHING FOR BHS IN THE LOCAL REGION}

We start by applying our model to the study of the innermost $250 \mathrm{pc}$ around Earth, following the work of Fender et al. (2013). In this study, the authors applied the Bondi formula to model accretion and estimated the number of visible sources through Monte Carlo simulations. They considered a few combinations of the values of the mean $\mathrm{BH}$ speed $\mu_{\mathrm{BH}}$ and of the suppression factor $\lambda$, obtaining a bound on the combination of these parameters. Here, we reproduce the same set-up to obtain a prediction for the number of detectable X-ray sources, verifying the result for the BHL model and comparing it to the predictions of the PR13 model.

\subsection{Our set-up}

First, we estimate the number $N^{\text {local }}$ of BHs present in the spherical region around Earth $(r=0)$ between $70 \mathrm{pc}<r<250 \mathrm{pc}$ (we exclude the innermost $70 \mathrm{pc}$ to account for the local void). As a rough estimate, we assume the fraction of $\mathrm{BHs}$ contained in this region to be equivalent to that of the progenitor stars. The total stellar mass in the Milky Way is $M_{\mathrm{MW}}^{*}=6 \times 10^{10} \mathrm{M}_{\odot}$ (Licquia \& Newman 2015), while the mass density of stars in the local region is around $7 \times 10^{-2} \mathrm{M}_{\odot} \mathrm{pc}^{-3}$ (McKee, Parravano \& Hollenbach 2015). Assuming a total of $10^{8} \mathrm{BHs}$ in the galaxy, we obtain

$N^{\text {local }}=10^{8} \frac{M_{\text {local }}^{*}}{M_{\mathrm{MW}}^{*}} \approx 7 \times 10^{3}$

While an accurate estimate of the number of BHs in the local region is beyond the scope of this work, we should notice that we are not taking into account the larger scale height of the $\mathrm{BH}$ population distribution with respect to the stellar one (a consequence of the natal kicks), which would result in a reduced local density. Our guess is thus likely overestimating the true number of $\mathrm{BHs}$ by a factor of $\mathcal{O}(1)$.

We assume a uniform spatial distribution, a normal mass distribution and an $\mathrm{MB}$ velocity distribution of average $\mu_{\mathrm{BH}}$, as described in the previous section. As for the interstellar medium, we assume two types of molecular clouds to be present, each type with uniform density. These are (a) warm clouds, of number density $10^{2} \mathrm{~cm}^{-3}$, sound speed $0.9 \mathrm{~km} \mathrm{~s}^{-1}$, and filling factor $5 \times 10^{-2}$, and (b) cold clouds, of number density $10^{3} \mathrm{~cm}^{-3}$, sound speed $0.6 \mathrm{~km} \mathrm{~s}^{-1}$, and filling factor $5 \times 10^{-3}$ (Fender et al. 2013). This combination results in an average density $n_{\mathrm{avg}}=10 \mathrm{~cm}^{-3}$. The integral in equation (9) becomes

$N^{\text {sources }}=\sum_{\text {warm, cold }} N^{\text {clouds }} \int_{\phi>\phi^{*}} P\left(\mathrm{v}_{\mathrm{BH}}\right) P(M) P(r) \mathrm{dv}_{\mathrm{BH}} \mathrm{d} M \mathrm{~d} r$.

Here, $N_{\text {clouds }}$ indicates the number of BHs we expect to find in each type of cloud

$N^{\text {clouds }}=f_{\text {clouds }} N^{\text {local }}$,

where $f_{\text {clouds }}$ is the associated filling factor. We obtain $N_{\text {clouds }} \approx 30$ in the cold clouds and $N_{\text {clouds }} \approx 300$ in the warm ones.

Finally, the bolometric fraction of luminosity in the X-ray band is set to 0.3 .

\subsection{Results}

Let us first consider the X-ray flux associated with a generic isolated $\mathrm{BH}$ located in the local region and accreting gas from a molecular cloud as a function of the BH speed. In Fig. 2, we show this flux for both the PR13 accretion scenario and the conventional modelling based on the BHL formalism, the latter suppressed by a factor of $\lambda=0.01$. The two coloured bands correspond to the two densities considered for molecular clouds. The width of the band is obtained considering distances between 70 and $250 \mathrm{pc}$ and masses between 5 and 11 solar masses. We show for reference the detection threshold associated with the fourth INTEGRAL IBIS/ISGRI soft gammaray survey catalogue (17-100 keV; Bird et al. 2009), which is 


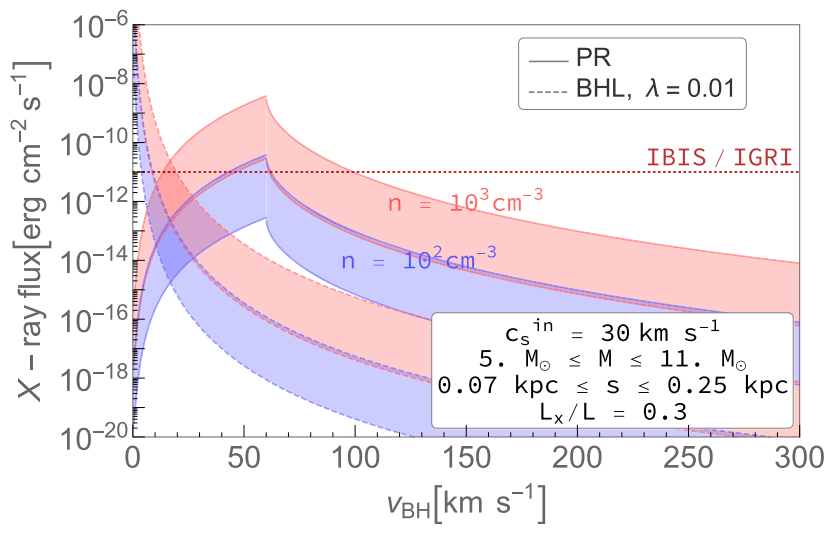

Figure 2. X-ray flux from $\mathrm{BHs}$ in the local region. We show the X-ray flux associated with a BH accreting from a molecular cloud in the local region, as a function of the relative velocity with respect to the gas cloud. The coloured bands describe the two types of clouds considered and their width corresponds to a range of $\mathrm{BH}$ masses and distances. We show the fluxes for the Park-Ricotti and the suppressed $(\lambda=0.01)$ BHL models. As a reference, we show the detection threshold associated with the IBIS/IGRI survey (Bird et al. 2009).

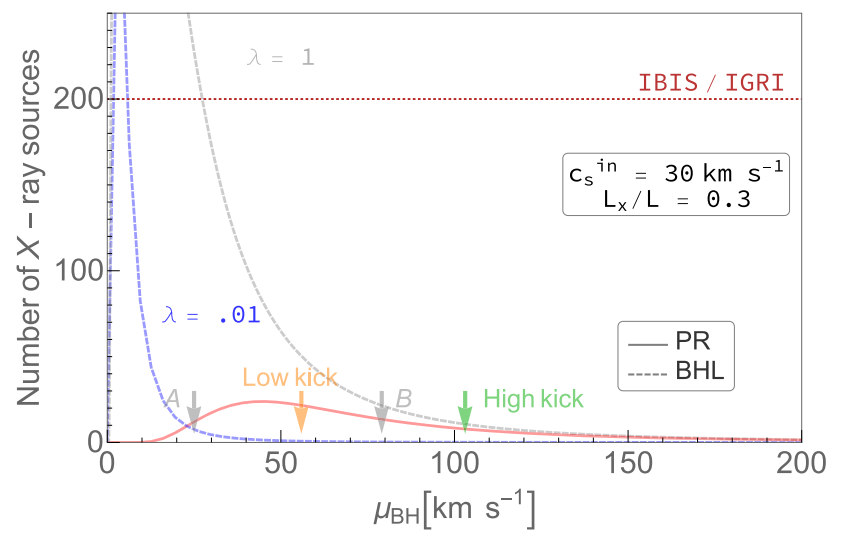

Figure 3. Total number of X-ray sources in the local region (full sky, $d<$ $250 \mathrm{pc}$ ). We show the expected number of BH observed in the X-ray as a function of the average BH speed according to (a) the PR13 model (red, solid), (b) the BHL model with $\lambda=1$ (grey, dashed), and (c) the BHL model with $\lambda=0.01$ (blue, dashed).

approximately $10^{-11} \mathrm{erg} \mathrm{cm}^{-2} \mathrm{~s}^{-1}$. We want to emphasize once again the distinct behaviour associated with the two accretion scenarios. In the Bondi picture, the slower sources are very luminous. This is particularly relevant in this context, since velocities in the local region are expected to be on the low end of the range shown in this figure: the unsuppressed BHL scenario predicts a huge number of sources (see Fig. 3) and can easily overshoot the bound. As a consequence of the introduction of a suppression factor, only very slow sources are expected to be visible. This is no longer true when the PR13 scenario is considered, since it naturally predicts a flux suppression for the slower sources. Instead, the population of BHs emerging above threshold and showing up in the X-ray sky will present relatively high speeds (around $50 \mathrm{~km} \mathrm{~s}^{-1}$ ). Furthermore, we can notice how, based on the Bondi picture, we expect to detect $\mathrm{BHs}$ in both types of clouds. According to the PR13 model, however, only BHs located in the denser clouds can be detected.

Following the procedure described in the previous section, we can now integrate the distributions that characterize the $\mathrm{BH}$ population to obtain the number of sources visible in the X-ray sky with the IBIS/IGRI survey. In Fig. 3, we show the dependence of number of sources on the average speed $\mu_{\mathrm{BH}}$. This prediction is obtained for three accretion scenarios: (a) the PR13 model; (b) the BHL model with suppression factor of $\lambda=0.01$; and (c) the BHL model with perfect efficiency $\lambda=1$. We indicate with orange and green arrows the reference values, given by the 'low kick' the 'high kick' scenarios, considering a velocity dispersion of the progenitor stars of $15 \mathrm{~km} \mathrm{~s}^{-1}$ (corresponding to an average speed of $\approx 25 \mathrm{~km} \mathrm{~s}^{-1}$ ). For comparison, the average speeds corresponding to the 'no kick' and 'high kick' $\left(\mu_{\text {kick }}=75 \mathrm{~km} \mathrm{~s}^{-1}\right)$ scenarios considered in Fender et al. (2013) are also shown and indicated with $\mathrm{A}$ and $\mathrm{B}$, respectively.

Our results can be summarized as follows:

(i) As far as the BHL scenario is concerned, we notice that the number of sources can easily overshoot the observational constraints in absence of a significant natal kick. We also point out that our findings are in broad agreement with the ones reported in Fender et al. (2013): i.e. a few tens of visible sources in scenario A (no kick, $\lambda=0.01)$.

(ii) In the PR13 scenario, the introduction of a suppression factor is not necessary. While being compatible with the experimental bound, our model nevertheless predicts a significant number of bright sources. This prediction corresponds to a population of $\sim 10-30$ accreting isolated BHs in the existing catalogues, taking as reference the 'no kick' and 'high kick' scenarios. We will discuss in more detail the consequences of this result in the next sections, and compare this prediction with the one associated with the Central Molecular Zone.

\section{SEARCHING FOR BHS IN THE CENTRAL MOLECULAR ZONE}

In this section, we turn our attention to the inner part of the Galactic bulge. This region is a particularly promising target for $\mathrm{BH}$ searches because of the presence of a large reservoir of molecular gas called central molecular zone (CMZ), a cloud complex with asymmetric shape that extends up to $\simeq 200 \mathrm{pc}$ away from the Galactic Centre. The CMZ has been the object of extensive multiwavelength observational campaigns over the years, aimed at characterizing its structure and physical properties, including star formation rate [see for instance Kruijssen et al. (2019) and references therein for a recent analysis]. The abundance of gas in the form of giant molecular clouds and the location close to the gravitational centre of the Galaxy clearly make it the ideal target for our study.

\subsection{Our set-up}

To model the CMZ, we employ a simplified version of the model by Ferrière, Gillard \& Jean (2007). We describe it as a cylindrical region of half height $15 \mathrm{pc}$ and radius $160 \mathrm{pc}$. The number of BHs contained in this region is of course very uncertain, but we can make a naive order-of-magnitude estimate, as follows. We assume the BHs to be generated following the distribution of stars in the Galaxy. Here, we are interested in the bulge component, which accounts for around 15 percent of the total and can be modelled with a spherical exponential with scale radius $R_{\text {bulge }}=120$ pc (Sofue 2013). Integrating the bulge spherical exponential distribution over the $\mathrm{CMZ}$ volume gives us the fraction of $\mathrm{ABHs}$ born in this region: around 2 per cent of the bulge component. Assuming a total of $10^{8}$ $\mathrm{BHs}$ present in the whole Galaxy, this corresponds to $3 \times 10^{5} \mathrm{BHs}$. We must however take into account that, due to the large initial natal kicks, the initial spatial distribution is modified (Tsuna et al. 


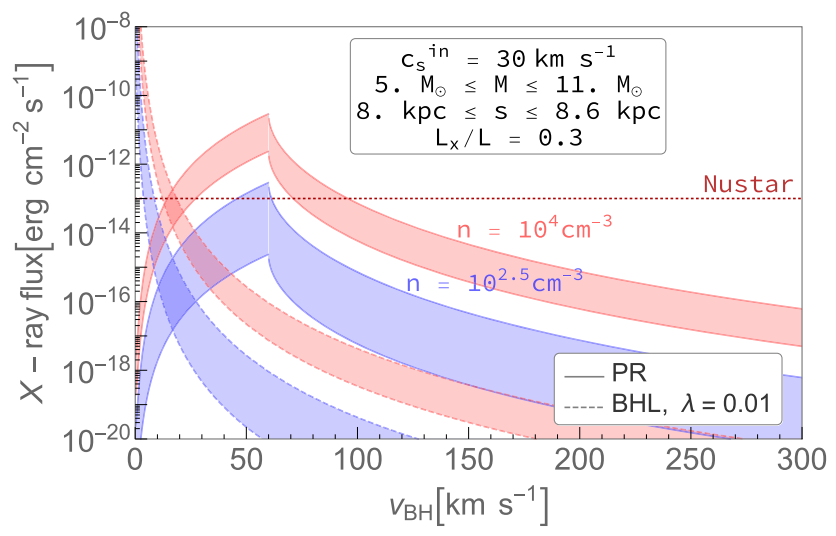

Figure 4. X-ray flux from BHs in the CMZ. We show the X-ray flux associated with a BH orbiting in the Central Molecular Zone molecular cloud complex for different values of the relative velocity with respect to the gas cloud, and the gas density in the cloud. We consider the Park-Ricotti and the BHL formalism. As a reference, we show the detection threshold associated with the NuSTAR Galactic Centre survey (Hong et al. 2016).

2018). We performed a simulation of the evolution in the Galactic potential (Irrgang et al. 2013, model II) of 1000 BHs. These are initially distributed uniformly in the $\mathrm{CMZ}$ region with an average speed of $130 \mathrm{~km} \mathrm{~s}^{-1}$ and given an average natal kick of $75 \mathrm{~km} \mathrm{~s}^{-1}$. The simulation shows that only $\approx 25$ percent of the $\mathrm{BHs}$ remain in the region: we observe in particular a spreading in the direction perpendicular to the Galactic plane. On the other hand, we do expect some BHs born outside of the $\mathrm{CMZ}$ to enter the region under the effect of the gravitational potential. However, these objects cross the $\mathrm{CMZ}$ close to the periastron of their orbit, with very large velocities and hence suppressed accretion rates. We can therefore neglect the latter effect. However, the former is significant and we take it into account. We thus update our naive estimate to $N^{\mathrm{CMZ}} \approx 7.5 \times 10^{4}$.

The molecular clouds in this region are known to be denser than the Galactic average. We assume an average number density of molecular hydrogen of $n_{\text {avg }}=150 \mathrm{~cm}^{-3}$ (Ferrière et al. 2007) and consider two types of clouds: warm, less dense clouds, and cold, denser ones. The warm clouds are of secondary importance, given the large distance from Earth (see Fig. 4). We set their density to $n=10^{2.5} \mathrm{~cm}^{-3}$ and consider a filling factor of 14 per cent (Ferrière et al. 2007). Regarding the cold clouds, we choose to adopt a more refined modelling based on a power-law density distribution between $n_{\min }$ and $n_{\max }$ :

$P(n) \propto n^{-\beta}$.

Based on Ferrière et al. (2007), we set $n_{\min }=10^{3.5} \mathrm{~cm}^{-3}$ and $n_{\max }=10^{5} \mathrm{~cm}^{-3}$, while we treat $\beta$ as a free parameter. The filling factor for the cold clouds is then obtained by requiring $n_{\text {avg }}=150 \mathrm{~cm}^{-3}$. For a reference value of $\beta=2.4$ (He, Ricotti \& Geen 2019, 2020), we obtain a filling factor of $\approx 1$ per cent and an average cloud density of $\approx 8 \times 10^{3}$.

The temperature of the clouds enters the accretion rate through the sound speed $c_{\mathrm{s}}$. As we mentioned before, this variable has little impact on the predictions and we set it to $1 \mathrm{~km} \mathrm{~s}^{-1}$.

Regarding the speed distribution, the average speed $\mu_{\mathrm{BH}}$ is treated as a free parameter. Nevertheless, we can make some estimates of reasonable values. The most recent observations suggest a velocity dispersion of the central bulge stars of average $\mu_{\text {bulge }} \approx 130 \mathrm{~km} \mathrm{~s}^{-1}$ (Brown et al. 2018; Sanders et al. 2019). This gives, for reference, $\mu_{v}=140 \mathrm{~km} \mathrm{~s}^{-1}$ in the 'low kick' scenario and $\mu_{v}=160 \mathrm{~km} \mathrm{~s}^{-1}$ in the 'high kick' scenario.
Finally, we approximate the distance distribution with a delta peaked at $8.3 \mathrm{kpc}$ and we assume the normal mass distribution discussed in Section 5.

The integral to be computed becomes

$$
\begin{aligned}
N^{\text {sources }}= & N_{\text {warm }}^{\text {clouds }} \int_{\phi>\phi^{*}} P\left(\mathrm{v}_{\mathrm{BH}}\right) P(M) \mathrm{dv}_{\mathrm{BH}} \mathrm{d} M \\
& +N_{\text {cold }}^{\text {clouds }} \int_{\phi>\phi^{*}} P\left(\mathrm{v}_{\mathrm{BH}}\right) P(M) P(n) \mathrm{dv}_{\mathrm{BH}} \mathrm{d} M \mathrm{~d} n .
\end{aligned}
$$

The expected number of BHs in the clouds is obtained as

$N^{\text {clouds }}=f_{\text {clouds }} N^{\mathrm{CMZ}}$,

where $f_{\text {clouds }}$ is the associated filling factor.

In summary, the set of relevant parameters that play a key role in our calculation are (1) the average $\mathrm{BH}$ speed, $\mu_{\mathrm{BH}}$; (2) the slope of the power-law density distribution, $\beta ;(3)$ the sound speed within the ionized region around the $\mathrm{BH}, c_{s}{ }^{\text {in }}$; (4) the fraction of bolometric luminosity irradiated in the hard X-ray band $L_{\mathrm{X}} / L ;(5)$ the number of BHs in the CMZ, $N^{\mathrm{CMZ}}$.

\subsection{Results}

We start by considering the X-ray flux associated with a generic isolated BH located in the Central Molecular Zone and accreting gas from a molecular cloud as a function of the speed and of the molecular cloud density. We show this observable in Fig. 4 for the two accretion scenarios discussed above. As a reference, we show the detection threshold associated with the NuSTAR Galactic Centre survey in the 3-40 keV band (Hong et al. 2016). The same trends highlighted in Section 6.2 can be noticed in this plot: In particular, we point out that, if the cloud density is high enough, there is a wide range of $\mathrm{BH}$ speed associated with emission above threshold within the PR13 formalism. On the other hand, no sources are expected to be detected in the lower density clouds.

With the X-ray flux for a CMZ source at hand, we apply the procedure described in the previous sections, and compute the number of X-ray sources associated with accreting BHs in this region. The results are shown in Figs 5 and 6, giving an impression of the impact of the different free parameters on this key observable. In particular, we show in panel (a) of Fig. 5 the number of X-ray sources in the $\mathrm{CMZ}$ region as a function of the average speed of the $\mathrm{BH}$ population adopting both the BHL and the PR13 accretion models. We show the result for various choices of the $\beta$ parameter defining the density distribution, and for two choices of the $\lambda$ parameter in the BHL case. We can notice how, in this setting, the PR13 scenario predicts as many sources as the $\lambda=1 \mathrm{BHL}$, due to the fact that high speeds are prevalent among the $\mathrm{BH}$ population of the $\mathrm{CMZ}$. However, we have seen that the unsuppressed BHL scenario is excluded by previous studies on nearby compact objects and complementary studies focused on AGN populations as mentioned in Section 2.1. The comparison should therefore be made between the predictions of the PR13 model and the suppressed BHL model, also shown in figure for $\lambda=0.01$. Then, the PR13 model predicts a significantly greater number than those obtained assuming BHL accretion. (See also the work of Tsuna et al. (2018), in which $\mathcal{O}(1)$ sources were predicted in the Galactic centre using BHL accretion.) In panel (b), we focus on the dependence of the same observable with respect to the fraction of the bolometric luminosity that is radiated in the X-ray band of interest. In Fig. 6, we consider the differential contribution of 

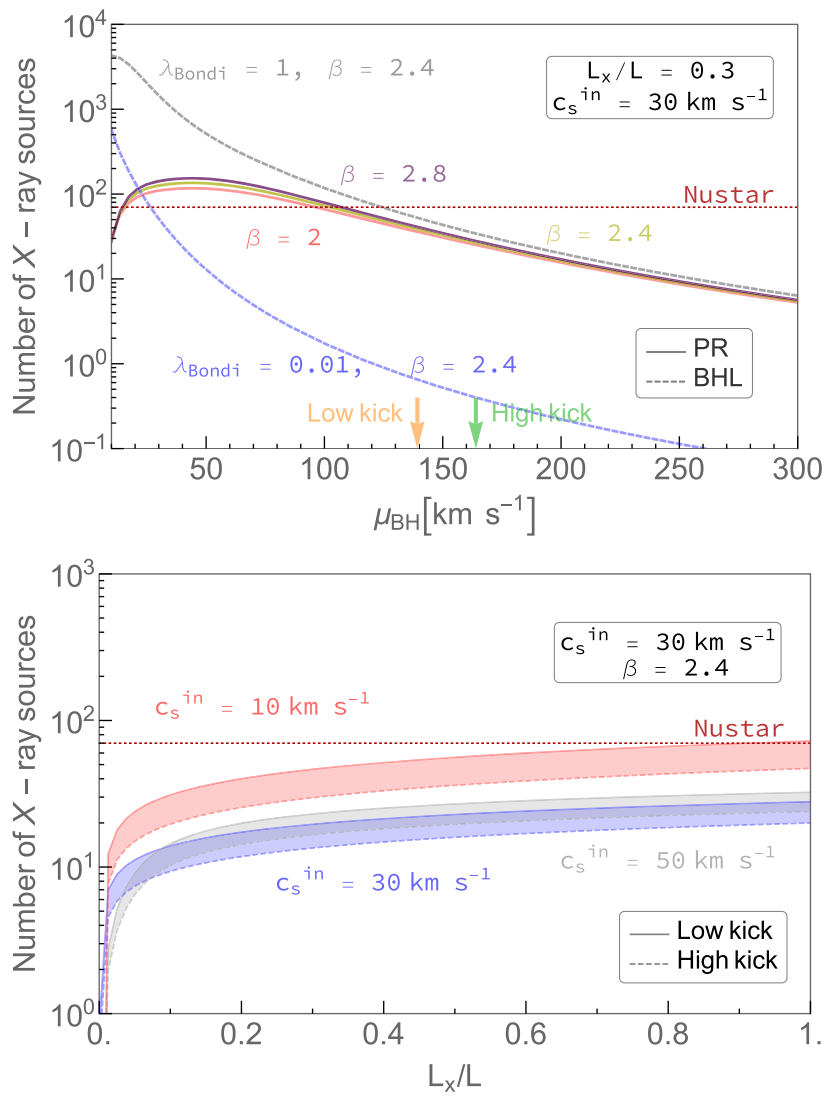

Figure 5. Parametric study of the number of X-ray sources in the CMZ. (a) Number of X-ray sources as a function of the average speed. (b) Number of $\mathrm{X}$-ray sources as a function of the bolometric luminosity that is radiated in the $\mathrm{X}$-ray band of interest. We compare this with the number of sources detected by NuSTAR in the CMZ region (Hong et al. 2016).

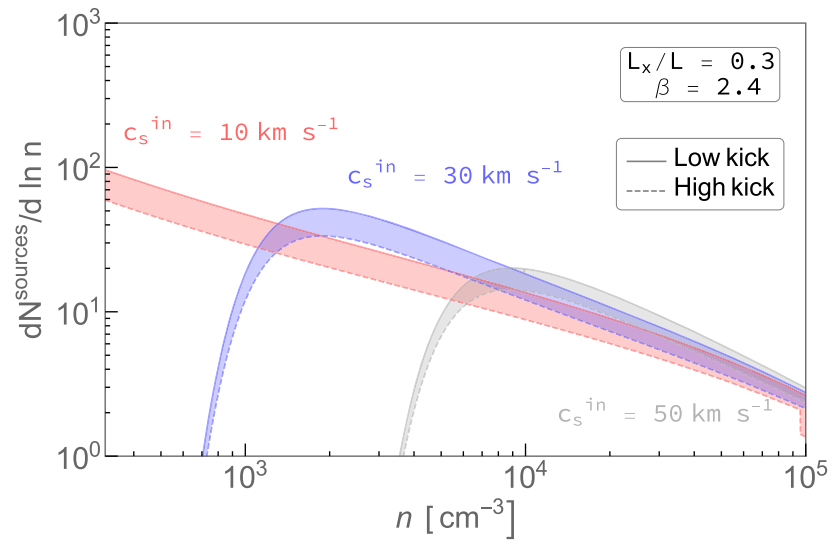

Figure 6. Distribution of bright $\mathrm{X}$-ray sources as a function of clump density. We show the log-differential number of sources detected by NuSTAR as a function of the clump density. A power-law distribution for the clump density is assumed.

the clumps of different density, again for different reference values of the ionized sound speed in the $\mathrm{BH}$ vicinity $c_{s}^{\text {in }}$.

We can conclude from the results visualized in Figs 5 and 6 that, despite the relevant uncertainties associated with the modelling of such a complex set-up (and despite the existence of a threshold effect due to the peak in the accretion rate), the prediction of few tens of

sources is solid with respect to these uncertainties. In particular, we notice from panel (a) that a number of bright X-ray sources comprised between 10 and 20 is expected for our reference value of $c_{s}^{\text {in }}$ and for $\mu_{\mathrm{BH}}=150 \mathrm{~km} \mathrm{~s}^{-1}$. As far as the speed distribution is concerned, we remark that the two reference values we have chosen to bracket the uncertainty both lead to similar predictions, while large deviations from this range would require to assume unrealistically high speeds. The same line of thought applies to the slope associated with the clump density distribution. From panel (b), we also notice a lower number of sources may only arise if we were to assume either a very high temperature in the ionized region, or else a very low fraction of bolometric luminosity radiated in the X-ray band. We remark, however, that the prediction scales linearly with the expected number of BHs in the region, $N^{\mathrm{CMZ}}$, which carries a large uncertainty.

In summary, the PR 13 model (together with our rather conservative assumptions regarding the $\mathrm{X}$-ray emission) leads to the prediction of a significant number of $\mathrm{X}$-ray sources in the $\mathrm{CMZ}$ region associated with isolated BHs accreting from molecular clouds. The total number of sources is of the same order of magnitude as the one predicted in the local region and discussed in the previous section. However, while the previous section was dealing with a full-sky analysis, in this case we are focusing on a specific region of interest (ROI) with small angular extent, which is ideal for multiwavelength observational campaigns. We recall that NuSTAR has detected 70 hard X-ray sources in this region interest. The nature of most of the sources in the catalogue in not precisely known, although a significant population of cataclysmic variables and X-ray binaries is expected. Our finding seems to suggest the need of a careful data analysis, in order to identify the possible presence of a relevant population of isolated accreting BHs in the already existing data. Following this line of thought, and in the prospect of a discovery, a multiwavelength analysis is compelling, so we will turn our attention to the radio domain in the next section.

\section{MULTIWAVELENGTH PROSPECTS FOR THE SQUARE KILOMETER ARRAY}

In the previous section, we have shown that, in a wide portion of the parameter space associated with our problem, a large number of bright X-ray sources are expected in the Galactic Centre region. However, in order to pinpoint a source as an accreting BH, a careful multiwavelength study has to be performed. The GHz radio band is particularly interesting in this context. As in previous works (Gaggero et al. 2017; Manshanden et al. 2019), the empirical scaling between X-ray and radio flux (Plotkin et al. 2012) discussed in detail in Section 3 (equation 8) is employed to obtain the radio flux.

A remarkable increase in the sensitivity is expected in the radio domain over the coming decade, thanks to the development of the Square Kilometer Array (SKA) project. This experiment has a huge potential towards shedding light on key problems of fundamental physics, cosmology, and astrophysics (Weltman et al. 2020). Here, we focus on the discovery potential of the population of astrophysical $\mathrm{BHs}$ in the $\mathrm{CMZ}$ at $1.4 \mathrm{GHz}$ band, and provide estimates of the number of potentially detectable sources by the SKA1-MID facility. Assuming gain $G=15 \mathrm{~K} / \mathrm{Jy}$, receiver temperature $T_{\mathrm{rx}}=25 \mathrm{~K}$, sky temperature towards the Galactic Centre $T_{\text {sky }}=70 \mathrm{~K}$, and bandwidth $\delta v=770 \mathrm{MHz}$ (as in Calore et al. 2016), we obtain an instrumental detection sensitivity of $2.7 \mu \mathrm{Jy}$ for a one-hour exposure. In the following, we assume an optimistic $1000 \mathrm{~h}$ exposure time and consistently adopt a potential detection threshold of $85 \mathrm{nJy}$.

In Fig. 7, we show the radio flux associated with a generic $\mathrm{BH}$ in the CMZ cloud, and compare it with the prospective SKA sensitivity and with the threshold of the VLA catalogue (Lazio \& Cordes 2008). 


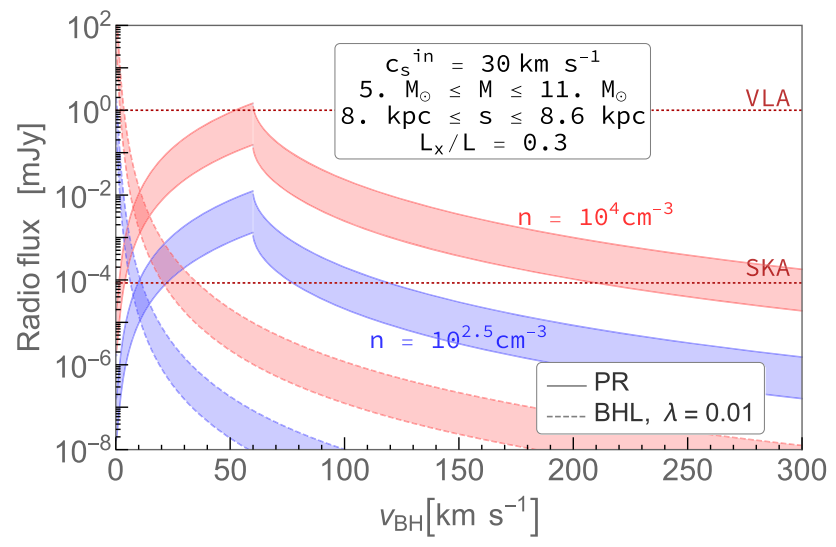

Figure 7. Radio flux from BHs in the CMZ. We show the radio flux for a generic $\mathrm{BH}$ in the CMZ. The present VLA sensitivity and the prospective sensitivity for SKA are shown.

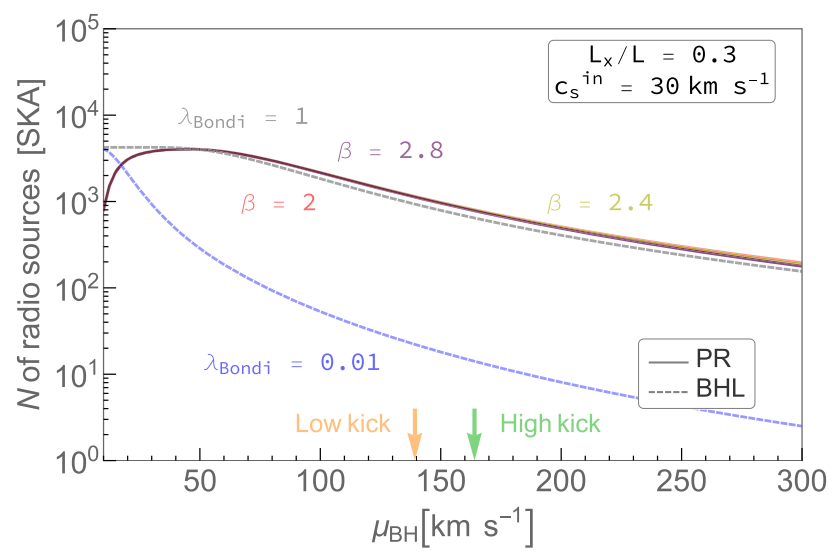

Figure 8. Radio prediction (I). We show the number of radio sources expected from the CMZ as a function of the number density of gas in molecular clouds, for different choices of the relevant parameters, obtained without taking into account the information from the X-ray band. The combination of this prediction with the X-ray bound is presented in Fig. 9.

Focusing on the PR13 scenario, we can conclude from this plot that, while we do would not expect to detect any isolated BH given the VLA sensitivity, SKA would be able to unveil a huge population of isolated $\mathrm{BH}$ in the Galactic Centre. In fact, most of the BHs accreting from the cold clouds would emit above the SKA threshold.

We show our prediction for the number of radio sources detectable with SKA in Figs 8 and 9, obtained with the same set-up described in Section 7.1. In particular, in Fig. 8, we show the number of radio sources detectable by SKA as a function of the BH speed, for various choices of the parameters discussed in the previous section. Our model predicts thousands of visible sources for the two reference values we have chosen to bracket the uncertainty on the speed distribution.

Let us now widen our perspective and promote $N^{\mathrm{CMZ}}$, the total number of $\mathrm{BHs}$ in the $\mathrm{CMZ}$, to a free parameter (We recall that this parameter encloses much of the uncertainties of our model). In Fig. 9, we show the number of bright radio sources in the $\left(\mu_{\mathrm{BH}}\right.$, $N^{\mathrm{CMZ}}$ ) space. In this parameter space, we may use the comparison with NuSTAR observations discussed in the previous section to obtain a bound: in particular, we can identify an upper limit on the number of BHs present in the $\mathrm{CMZ}$, by requiring not to overshoot the number of sources observed by that experiment. In the portion of

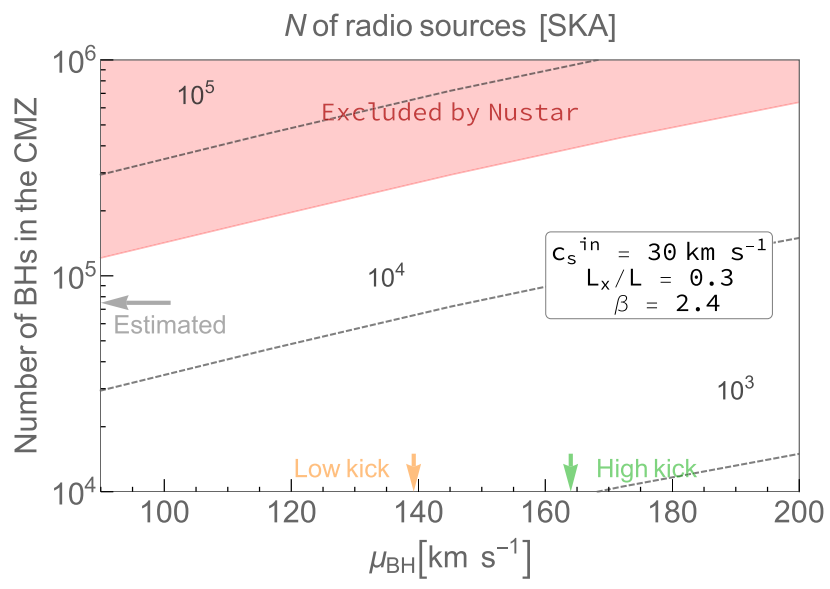

Figure 9. Radio prediction (II). We show the contour lines for the number of sources potentially detectable by the Square Kilometer Array as a function of cloud density and total number of BHs in the $\mathrm{CMZ}$ clouds. The red region corresponds to the parameter space region excluded by NuSTAR observations. The grey arrow indicates the estimate of the number of BHs in the $\mathrm{CMZ}$ region used as reference in the previous section.

parameter space compatible with the NuSTAR bound, $\mathcal{O}\left(10^{4}\right)$ radio point sources could be detected by SKA. Such order of magnitude estimate is solid with respect to the uncertainties of the remaining parameters of the model.

Of course, this prediction relies on the assumption that the FP scaling between radio and X-ray emission holds, which in turn requires assuming the emission of a jet. While this is not disproven, we are neither able to assert with certainty that every accreting isolated $\mathrm{BH}$ will emit a jet. If no jet is emitted, we would expect a lower radio flux. It is interesting to assess the prospects in case of a lower radio emission with respect to the FP prediction. The SKA sensitivity we consider here corresponds to a flux about $\approx 10^{8}$ times lower than the one detectable with the NuSTAR sensitivity. The FP predicts a luminosity ratio $L_{\mathrm{R}} / L_{\mathrm{X}}$ of around $10^{-6}$ at peak luminosity. Therefore, 1 percent of the radio luminosity predicted by the FP scaling would be sufficient for all NuSTAR isolated BH sources to have a radio counterpart detectable by SKA. The most luminous sources would still be visible if the radio flux is 0.01 per cent of that predicted by the FP scaling.

We can therefore conclude that the radio searches for isolated BHs in the CMZ constitute a very promising science case for SKA.

\section{DISCUSSION}

\subsection{Comments on the parameter space}

We have concluded that our predictions for the $\mathrm{CMZ}$ are stable in a very wide region of the parameter space of the model. However, we should note that we made a number of assumptions regarding the distribution of $\mathrm{BHs}$ in the $\mathrm{CMZ}$. On the one hand, as we already discussed, our estimate of the total number of BHs in the CMZ is obtained with a simple model for the initial distribution and subsequent orbit evolution. Furthermore, we have assumed that both the BHs and the clouds are uniformly distributed in the CMZ. The presence of a positive or negative correlation between the two distributions could in principle impact the results. We further assumed a specific model for the cloud density distribution. These simplifications eventually affect the number of BHs in the clouds, 
and therefore the final predictions on the number of sources, which scale linearly with this quantity.

\subsection{The accretion scenario}

We have shown in Section 6 that, based on local observations, the PR13 accretion model does not require the introduction of a suppression factor $\lambda$. However, since the PR13 model relies on the BHL model to describe accretion within the ionized region (see Section 2), one may wonder whether the BHL suppression factor should be introduced at that stage. With respect to this, we should take into account that this suppression factor is usually associated with a very strong outflow of matter from within the Bondi radius, associated with a fraction of $1-\lambda$ of the total matter initially accreted. Such a large outflow is not observed in the simulations of Park \& Ricotti (2013), where most matter crossing the Bondi radius within the ionized region ultimately reaches the $\mathrm{BH}$. We conclude from this that introducing a suppression factor in the expression would not be physically justified. We remark that the simulations considered here represent the state of the art in the field. However, some physical ingredients are still not captured: we mention in particular the role of magnetic fields. Future works will assess the potential impact of a full magnetohydrodynamical treatment in this context.

\subsection{The emission mechanism and spectrum}

In Section 7.1, we also assume that $\sim 30$ per cent of the total bolometric luminosity of all our isolated BHs falls within the observable X-ray band $-L_{\mathrm{X}}=0.3 L_{\mathrm{bol}}$. This assumption was based on previous estimates, for example those presented by Fender et al. (2013). However, it is worth assessing the accuracy and precision of this assumption. We assumed a flat radio spectrum $\left(L_{v} \propto v^{0}\right)$, and then an additional inverted power law in the X-ray band. The assumption that 30 per cent of the observed bolometric luminosity falls in the X-ray band depends on the spectral index of this additional power law, $\alpha$, the high-energy cut-off of the power law, $E_{\text {cut }}$, the absorbing hydrogen column density along the line of sight, $N_{\mathrm{H}}$, and the observing energy band (which we conservatively adopted as $3-$ $40 \mathrm{keV}$ for the NuSTAR X-ray telescope). Inefficiently radiating Galactic BH-XRBs are dominated by power-law emission with a typical range for the spectral index given by $0.6 \leq \alpha \leq 1.0$ (or a photon index of $1.6 \leq \Gamma \leq 2$ in common X-ray astronomy parlance). However, the power-law spectra of Galactic BH-XRBs can become softer in intermediate spectral states, or very quiescent states, so we can extend this out to an upper bound of $\alpha \leq 1.4$ or $\Gamma \leq 2.4$. Adopting a rough estimate of the jet break frequency of $\sim 10^{14} \mathrm{~Hz}$ (infrared frequencies; Russell et al. 2013), a typical highenergy cut-off in the $\mathrm{X}$-ray of $100 \mathrm{keV}$, and a high hydrogen column density of $10^{23} \mathrm{~cm}^{-2}$, we calculate that $L_{\mathrm{X}} \sim(0.24-0.35) L_{\mathrm{bol}}$. The corresponding unabsorbed range of fractional luminosities is then $0.27-0.46 L_{\mathrm{bol}}$. These predictions, however, depend strongly on the cut-off energy. For example, just reducing the cut-off energy from 100 to $80 \mathrm{keV}$ can increase the fractional luminosity to $\sim 60-70$ per cent. Therefore, our assumption that $L_{\mathrm{X}} \sim 0.3 L_{\mathrm{bol}}$ is rather conservative, even if we assume strong absorption along the line of sight. It is also based upon a relatively hard power-law spectrum in the X-ray band. Nevertheless, we have shown in Section 7 that our predictions are solid with respect to variations of this parameter, even for values as large as 70 per cent.

For the radio flux calculations through the Fundamental Plane of Black Hole Activity (FP; Merloni et al. 2003; Falcke et al. 2004;
Plotkin et al. 2012) we had to assume the presence of a jet emitting in the $\mathrm{GHz}$ radio band, following Fender et al. (2013), Gaggero et al. (2017), and Manshanden et al. (2019). This crucial assumption is motivated by results of Fender (2001), which established that all highly sub-Eddington accreting BHs in X-ray binary systems are accompanied by such radio emission.

Further emission could come from other types of outflow that are less collimated or relativistic. Such alternative emission could constitute a lower limit on the expected radio emission, which would be of particular interest in the absence of a jet. Tsuna \& Kawanaka (2019) assumed a spherically symmetric outflow based on losing a fraction $(1-\lambda)$ of the accreting matter before reaching the $\mathrm{BH}$, where $\lambda$ is the suppression factor. Assuming a power-law radial dependence of the accretion rate, they used the escape velocity to conservatively estimate the power associated with such an outflow. It should be noted that this radial power-law assumption (with slope larger than 1) implies that most matter would be lost close to the $\mathrm{BH}$, which requires a powerful outflow. The shock from the collision of this outflow with the ISM could accelerate non-thermal electrons through diffusive shock acceleration and subsequently produce radiosynchrotron emission. The precise emission profile will be highly dependent on the fraction of energy going into the electrons and the magnetic field.

However, as highlighted above, such a relevant outflow is not observed in the simulations presented in Park \& Ricotti (2013) and hence this scenario is hard to unify with the PR13 accretion model. Instead, it would be interesting to further investigate what type of emission could be associated with the shock on the boundary of the cometary region itself, which would likely require additional detailed simulations.

\subsection{Connection with PBH searches}

We now want to widen the perspective and mention that the search for isolated astrophysical black holes (ABHs) can be considered a relevant problem per se, and also crucial as a background in the context of the quest for primordial black holes (PBHs) in the Galactic environment [see for instance García-Bellido (2017) for a discussion of different multimessenger channels regarding PBH searches]. The potential of this observational channel in constraining the abundance of PBHs in the inner part of the Galaxy, with particular focus on the CMZ region, was first pointed out in Gaggero et al. (2017), within the framework of the BHL formalism. In Manshanden et al. (2019), the impact of the Park-Ricotti formalism was assessed in detail. The main result of that work is a strong upper bound on the abundance of PBHs (in the context of a non-clustered distribution of PBHs with monochromatic, lognormal, and power-law mass functions). The upper bound was set at the level of $10^{-3}$ of the dark matter in the form of PBHs, which would correspond to roughly $\sim 10^{8}$ objects in the Milky Way for a $10 \mathrm{M}_{\odot}$ reference mass. Hence, the astrophysical population (which is expected to include the same order of magnitude of compact objects) is expected to be an irreducible background that could play the role of an astrophysical floor associated with the quest for a sub-dominant population of PBHs of tens of solar masses.

\subsection{Outlook}

We have shown that a scenario based on the PR13 accretion model and our best estimate of the number of $\mathrm{BHs}$ in the $\mathrm{CMZ}$ naturally implies a large number of detectable $X$-ray and radio sources, for any reasonable choice of the other free parameters involved in the problem. 
This relevant result is highly suggestive that a clear identification of a population of isolated, accreting BHs in the Galactic Centre region - by means of the current or forthcoming generation of radio and X-ray experiments - may be around the corner.

\section{SUMMARY}

In this paper, we presented a comprehensive study of the constraints and prospects of detection of a population of isolated astrophysical BHs in the solar vicinity and near the Galactic Centre. We have adopted the Park-Ricotti (PR13) accretion model that takes into account radiation feedback and is backed up by hydrodynamic numerical simulations, and compared the results obtained within this scenario with the ones obtained by exploiting the well-known BHL formalism. We found that, within the PR13 model, a few bright $\mathrm{X}$-ray sources associated with isolated accreting $\mathrm{BHs}$ are expected in the solar vicinity $(R<250 \mathrm{pc})$, and a fraction of the sources listed in current catalogues could be associated with such objects. We performed an extended parametric study about the number of detectable sources in the vicinity of the Galactic Centre, with particular focus to the promising Central Molecular Zone region. In this region of interest, we found that, despite the large uncertainty associated with the free parameters involved in the calculation, the prediction of $\mathcal{O}(10)$ X-ray sources above the detection threshold associated with the NuSTAR catalogue is solid for any reasonable choice of the parameters under scrutiny. In particular, a number of bright X-ray sources comprised between 10 and 20 is expected for our reference value of the ionized sound speed $c_{s}^{\text {in }}=30 \mathrm{~km} \mathrm{~s}^{-1}$ and an average $\mathrm{BH}$ speed $\mu_{\mathrm{BH}}=150 \mathrm{~km} \mathrm{~s}^{-1}$. A multiwavelength analysis will be needed to clearly identify such a population. In this context, we pointed out promising prospects of detection for future generation experiments in the radio band, with particular reference to the Square Kilometre Array.

\section{ACKNOWLEDGEMENTS}

The work of DG and FS has received financial support through the Postdoctoral Junior Leader Fellowship Programme from la Caixa Banking Foundation (grant no. LCF/BQ/LI18/11630014).

DG was also supported by the Spanish Agencia Estatal de Investigación through the grants PGC2018-095161-B-I00, IFT Centro de Excelencia Severo Ochoa SEV-2016-0597, and Red Consolider MultiDark FPA2017-90566-REDC.

MR acknowledges support by NASA grant no. 80NSSC18K0527.

RMTC has been supported by NASA ADAP grant no. 80NSSC177K0515.

\section{DATA AVAILABILITY}

No new data were generated or analysed in support of this research.

\section{REFERENCES}

Baganoff F. K. et al., 2003, ApJ, 591, 891 (PR13)

Bird A. J. et al., 2009, ApJS, 186, 1

Blandford R. D., Begelman M. C., 1999, MNRAS, 303, L1

Bondi H., Hoyle F., 1944, MNRAS, 104, 273

Brown A. G. A. et al., 2018, A\&A, 616, A1

Calore F., Di Mauro M., Donato F., Hessels J. W. T., Weniger C., 2016, ApJ, 827,143

Caputo D. P., de Vries N., Patruno A., Portegies Zwart S., 2017, MNRAS, 468,4000
Corbel S., Fender R. P., Tzioumis A. K., Nowak M., McIntyre V., Durouchoux P., Sood R., 2000, A\&A, 359, 251

Corbel S., Nowak M. A., Fender R. P., Tzioumis A. K., Markoff S., 2003, A\&A, 400, 1007

Corbel S., Koerding E., Kaaret P., 2008, MNRAS, 389, 1697

Esin A. A., McClintock J. E., Narayan R., 1997, ApJ, 489, 865

Falcke H., Körding E., Markoff S., 2004, A\&A, 414, 895

Farr W. M., Sravan N., Cantrell A., Kreidberg L., Bailyn C. D., Mandel I., Kalogera V., 2011, ApJ, 741, 103

Fender R. P., 2001, MNRAS, 322, 31

Fender R. P., Maccarone T. J., Heywood I., 2013, MNRAS, 430, 1538

Ferrière K., Gillard W., Jean P., 2007, AAP, 467, 611

Gaggero D., Bertone G., Calore F., Connors R. M. T., Lovell M., Markoff S., Storm E., 2017, Phys. Rev. Lett., 118, 241101

Gallo E., Fender R. P., Pooley G. G., 2003, MNRAS, 344, 60

Gallo E. et al., 2014, MNRAS, 445, 290

García-Bellido J., 2017, J. Phys. Conf. Ser., 840, 012032

He C.-C., Ricotti M., Geen S., 2019, MNRAS, 489, 1880

He C.-C., Ricotti M., Geen S., 2020, MNRAS, 492, 4858

Hobbs G., Lorimer D. R., Lyne A. G., Kramer M., 2005, MNRAS, 360, 974

Hong J. et al., 2016, ApJ, 825, 132

Hoyle F., Lyttleton R. A., 1939, Proc. Cambridge Phil. Soc., 35, 405

Irrgang A., Wilcox B., Tucker E., Schiefelbein L., 2013, A\&A, 549, A137

Kruijssen J. M. D. et al., 2019, MNRAS, 484, 5734

Lazio T. J. W., Cordes J. M., 2008, ApJS, 174, 481

Licquia T. C., Newman J. A., 2015, ApJ, 806, 96

Maccarone T. J., 2005, MNRAS, 360, L30

Manshanden J., Gaggero D., Bertone G., Connors R. M. T., Ricotti M., 2019, JCAP, 1906, 026

Markoff S., Nowak M. A., Wilms J., 2005, ApJ, 635, 1203

McKee C. F., Parravano A., Hollenbach D. J., 2015, ApJ, 814, 13

Merloni A., Heinz S., di Matteo T., 2003, MNRAS, 345, 1057

Miller-Jones J. C. A., Jonker P. G., Maccarone T. J., Nelemans G., Calvelo D. E., 2011, ApJ, 739, L18

Narayan R., Yi I., 1994, ApJ, 428, L13

Özel F., Psaltis D., Narayan R., McClintock J. E., 2010, ApJ, 725, 1918

Park K., Ricotti M., 2011, ApJ, 739, 2

Park K., Ricotti M., 2012, ApJ, 747, 9

Park K., Ricotti M., 2013, ApJ, 767, 163

Pellegrini S., 2005, ApJ, 624, 155

Perna R., McDowell J., Menou K., Raymond J., Medvedev M. V., 2003, ApJ, 598,545

Plotkin R. M., Markoff S., Kelly B. C., Körding E., Anderson S. F., 2012, MNRAS, 419, 267

Plotkin R. M., Markoff S., Kelly B. C., Körding E., Anderson S. F., 2012, MNRAS, 419, 267

Plotkin R. M., Gallo E., Markoff S., Homan J., Jonker P. G., Miller-Jones J. C. A., Russell D. M., Drappeau S., 2015, MNRAS, 446, 4098

Russell D. M. et al., 2013, MNRAS, 429, 815

Samland M., 1998, ApJ, 496, 155

Sanders J. L., Smith L., Evans N. W., Lucas P., 2019, MNRAS, 487, 5188

Shapiro S. L., Teukolsky S. A., 1983, Black Holes, White Dwarfs, and Neutron Stars: The Physics of Compact Objects. Wiley, New York

Sofue Y., 2013, PASJ, 65, 118

Sugimura K., Ricotti M., 2020, MNRAS, 495, 2966

Tsuna D., Kawanaka N., 2019, MNRAS, 488, 2099

Tsuna D., Kawanaka N., Totani T., 2018, MNRAS, 477, 791

Weltman A., et al., 2020, PASA, 37, e002

Yuan F., Quataert E., Narayan R., 2003, ApJ, 598, 301

\section{APPENDIX: DERIVATION OF THE PARK-RICOTTI MODEL}

We briefly summarize how the Park-Ricotti accretion model is derived, following closely Park \& Ricotti $(2011,2012,2013)$. Assuming a one-dimensional steady flux, the jump conditions obtained by 
applying Euler's equations for mass and momentum conservation at the ionization front are as follows:

$$
\begin{aligned}
& \rho_{\text {in }} \mathrm{v}_{\text {in }}=\rho \mathrm{v}_{\mathrm{BH}}, \\
& \rho_{\text {in }}\left(\mathrm{v}_{\text {in }}^{2}+c_{\mathrm{s}, \text { in }}^{2}\right)=\rho\left(\mathrm{v}_{\mathrm{BH}}^{2}+c_{\mathrm{s}}^{2}\right),
\end{aligned}
$$

where to obtain the second equation we have further assumed an ideal gas. These equations have the following solutions:

$\rho_{\text {in }}=\rho_{\text {in }}^{ \pm} \equiv \rho \frac{\mathrm{v}_{\mathrm{BH}}^{2}+c_{\mathrm{s}}^{2} \pm \sqrt{\Delta}}{2 c_{\mathrm{s}, \text { in }}^{2}}, \quad \Delta \equiv\left(\mathrm{v}_{\mathrm{BH}}^{2}+c_{\mathrm{s}}^{2}\right)^{2}-4 \mathrm{v}_{\mathrm{BH}}^{2} c_{\mathrm{s}, \mathrm{in}}^{2}$

$\mathrm{v}_{\text {in }}=\frac{\rho}{\rho_{\text {in }}} \mathrm{v}_{\mathrm{BH}}$,

for $\mathrm{v}_{\mathrm{BH}} \leq \mathrm{v}_{\mathrm{D}}$ or $\mathrm{v}_{\mathrm{BH}} \geq \mathrm{v}_{\mathrm{R}}$, with $\mathrm{v}_{\mathrm{D}}$ and $\mathrm{v}_{\mathrm{R}}$ the roots of $\Delta$. Since typically $c_{\mathrm{s}, \text { in }} \sim \mathcal{O}(10) \mathrm{km} \mathrm{s}^{-1}$ while $c_{\mathrm{s} \text {, out }} \sim \mathcal{O}(1) \mathrm{km} \mathrm{s}^{-1}$, we have

$\mathrm{v}_{\mathrm{R}} \approx 2 c_{\mathrm{s}, \text { in }}$,

$\mathrm{v}_{\mathrm{D}} \approx \frac{c_{\mathrm{s}}^{2}}{2 c_{\mathrm{s}, \mathrm{in}}} \ll 1 \mathrm{~km} \mathrm{~s}^{-1}$.

In the high-velocity range, $\mathrm{v}_{\mathrm{BH}} \geq \mathrm{v}_{\mathrm{R}}$, the correct solution is given by $\rho_{\text {in }}^{-}$, while for $\mathrm{v}_{\mathrm{BH}} \leq \mathrm{v}_{\mathrm{D}}$, the front is best described by $\rho_{\text {in }}^{+}$(Park \& Ricotti 2013). In the intermediate-velocity range, $\mathrm{v}_{\mathrm{D}}<\mathrm{v}_{\mathrm{BH}}<\mathrm{v}_{R}$, no real common solution can be obtained for equations (A1) and (A2). Correspondingly, as velocity lowers below $\mathrm{v}_{R}$, the pressure wave building behind the ionization front detaches from it as a shock into the neutral material. Part of the flux of matter is deviated and the velocity of the gas beyond the shock is reduced to below $\mathrm{v}_{D}$ This implies on the one hand that equation (A1), stating the mass conservation through the front along the direction of displacement of the $\mathrm{BH}$, is no longer valid. On the other hand, since the velocity past the shock is now lower than $\mathrm{v}_{D}$, the jump conditions at the ionization front can be solved. One should now consider, however, two sets of jump conditions associated with the two fronts: the shock and the ionization front. Park and Ricotti instead observed through simulations that, in this regime, the velocity inside the ionized region is $\mathrm{v}_{\text {in }} \approx c_{\mathrm{s} \text {, in }}$. This relation, promoted to an equality, can be used together with equation (A2) to compute the density $\rho_{\text {in }}$. This way, for $\mathrm{v}_{D} \leq \mathrm{v}_{\mathrm{BH}} \leq \mathrm{v}_{R}$, we obtain

$$
\begin{aligned}
& \rho_{\text {in }}=\rho_{\text {in }}^{0} \equiv \rho \frac{\mathrm{v}_{\mathrm{BH}}^{2}+c_{\mathrm{s}}^{2}}{2 c_{\mathrm{s}, \text { in }}^{2}}, \\
& \mathrm{v}_{\text {in }}=c_{\mathrm{s}, \text { in }} .
\end{aligned}
$$

Thus we have in summary:

$\rho_{\text {in }}= \begin{cases}\rho_{\text {in }}^{-}, & \mathrm{v}_{\mathrm{BH}} \geq \mathrm{v}_{\mathrm{R}}, \\ \rho_{\text {in }}^{0}, & \mathrm{v}_{\mathrm{D}}<\mathrm{v}_{\mathrm{BH}}<\mathrm{v}_{\mathrm{R}}, \\ \rho_{\text {in }}^{+}, & \mathrm{v}_{\mathrm{BH}} \leq \mathrm{v}_{\mathrm{D}},\end{cases}$

and

$\mathrm{v}_{\text {in }}= \begin{cases}\frac{\rho}{\rho_{\text {in }}} \mathrm{v}_{\mathrm{BH}}, & \mathrm{v}_{\mathrm{BH}} \geq \mathrm{v}_{\mathrm{R}}, \\ c_{\mathrm{s}, \mathrm{in}}, & \mathrm{v}_{\mathrm{D}}<\mathrm{v}_{\mathrm{BH}}<\mathrm{v}_{\mathrm{R}}, \\ \frac{\rho}{\rho_{\text {in }}} \mathrm{v}_{\mathrm{BH}}, & \mathrm{v}_{\mathrm{BH}} \leq \mathrm{v}_{\mathrm{D}} .\end{cases}$

Plugging these equations back into equation (2) finally gives the desired accretion rate expressed in terms of the $\mathrm{BH}$ speed and the properties of the neutral medium it is moving through.

This paper has been typeset from a $\mathrm{T}_{\mathrm{E}} \mathrm{X} / \mathrm{E} \mathrm{T} \mathrm{E} \mathrm{X}$ file prepared by the author. 